\title{
Scaling Properties of Random Walks on Small-World Networks
}

\author{
E. Almaa: * \\ Department of Physics, University of Notre Dame, Notre Dame, Indiana 46556 \\ R. V. Kulkarni $\S \dagger$ \\ Department of Physics, University of California, Davis, California 95616 \\ D. Stroud \\ Department of Physics, The Ohio State University, Columbus, Ohio 43210
}

(Dated: November 5, 2018)

\begin{abstract}
Using both numerical simulations and scaling arguments, we study the behavior of a random walker on a one-dimensional small-world network. For the properties we study, we find that the random walk obeys a characteristic scaling form. These properties include the average number of distinct sites visited by the random walker, the mean-square displacement of the walker, and the distribution of first-return times. The scaling form has three characteristic time regimes. At short times, the walker does not see the small-world shortcuts and effectively probes an ordinary Euclidean network in $d$-dimensions. At intermediate times, the properties of the walker shows scaling behavior characteristic of an infinite small-world network. Finally, at long times, the finite size of the network becomes important, and many of the properties of the walker saturate. We propose general analytical forms for the scaling properties in all three regimes, and show that these analytical forms are consistent with our numerical simulations.
\end{abstract}

PACS numbers: 87.18.Sn, 05.10.-a, 05.40.-a, 05.50.+q

\section{INTRODUCTION}

The topological properties of real-world networks have been studied extensively. But an even more intriguing task, and a natural extension of these studies, is to understand how the network structure affects dynamics on the networks. 1] Most people have had the unfortunate experience of catching the flu (an example of disease spreading) or picking up a burning hot plate (an example of neural signal transmission). These phenomena are all examples of dynamics on the special kind of real-world networks that have been found to display "small-world" properties. Of these phenomena, the greatest attention thus far has been given to the study of disease spreading [see, e. g. [2, 3, 4, [5]]. It has even been suggested that the web of sexual contacts has a small-world structure [6, 7]. Other dynamical models that have been studied on complex networks include the Hodgkin-Huxley model [8], Boolean dynamics [9], and the generic synchronization of oscillators [10]. Extensive reviews can be found in Refs. 1, 2, 11].

In this paper, we will present results for a random walk on a small-world network (SWN, defined below). Such random walks may have several applications to real systems. For example, Scala et al 12 have argued that the conformation space of a lattice polymer has a small-world topology, and hence, that diffusion and random walks on

\footnotetext{
*Electronic address: Almaas.1@nd.edu

${ }^{\dagger}$ Electronic address: rahul@research.nj.nec.com

${ }^{\ddagger}$ Electronic address: stroud@mps.ohio-state.edu
}

such small-world networks might give insight into relaxation processes such as protein folding.

Much is known about random walks on both regular and random networks (see, e. g., 13, 14]). In addition, there have been several recent studies of random walks on SWN's 12, 15, 16, 17, 18, 19, 20, 21]. For example, Jasch and Blumen [17] and Lahtinen et. al [18] have studied the average number of distinct sites visited by a walker, and the probability that the walker is at the origin after $n$ steps in the limit that the network size $L \rightarrow \infty$ and, hence, the number of shortcuts $x \rightarrow \infty$. In the present work, we present results for the scaling behavior of such quantities as the mean-square displacement, the mean number of distinct sites covered during a random walk, the first-return time abd clarify the procedure for obtaining a scaling collapse for random walks on SWN's.

\section{THE RANDOM WALK}

In previous work 22, 23], we developed a simple probability function approach for the topological properties of complex networks generated according to the smallworld model [24, 25]. In this approach, we start from a one-dimensional regular network with periodic boundary conditions and $L=2 N$ nodes, each node being connected to its $2 k$ nearest neighbors. Hence, the "degree" of each node is $2 k$. Next, we add shortcut ends to each node according to a given degree distribution $\left(\mathcal{D}_{q}\right)$, by following the prescription of Refs. 26, 27]. In the present work we use a modified approach [23] by using the following degree distribution in one dimen- 
sion $($ with $k=1): \mathcal{D}_{q}=(1-p) \mathcal{P}_{(q)}(p)+p \mathcal{P}_{(q-1)}(p)$, where $\mathcal{P}_{q}(\lambda)=\exp (-\lambda) \lambda^{q} / q$ ! is the Poisson distribution. We then select pairs of shortcut ends at random and connect them to each other, thus creating a shortcut. This network-generating procedure (with the above $\mathcal{D}_{a}$ ) is equivalent to that outlined by Newman and Watts [25]. The quantity $k p$ is the probability that a given site has a shortcut. On average, there will be $x=k p L$ shortcuts in the network.

We carry out the random walk on a lattice (Polya walk) as follows: (i) There is only one walker on the small-world network at a time. (ii) The random walker is injected onto a randomly chosen site on the small-world network, a new site for each walker. We will call this site the "origin" of the walk. (iii) At each discrete time step, $t$, the walker will jump to a randomly chosen nearest neighbor of its current site $m$ with probability $1 / k(m)$. Here, $k(m)$ is the number of nearest neighbors of site $m$, i.e. the degree of node $m$. (iv) The random walker is allowed to wander the network for a time longer than the "saturation time" for the quantity studied, i.e. the time when that quantity approaches its limiting behavior. (v) We average over different random walkers and realizations of the small-world lattice until the results converge.

\section{SCALING BEHAVIOR}

\section{A. General Form}

In earlier work 22, 23], we demonstrated that the basic probability distribution $P(m \mid n ; L, p)$ (the probability that two sites separated by $n$ hops before the introduction of shortcuts has a minimal separation of $m$ hops when shortcuts are included) scales with $x$ in the limit of $p \ll 1$, for all choices of $x$. As a consequence, topological quantities derived from $P(m \mid n ; L, p)$ displays a scaling with $x=p L$ in the same limit.

We will first state our main result for the scaling of the random walk in general, and then consider specific examples. Let $O(p, L, t)$ be some measurable quantity for the random walk on an SWN, which saturates to a finite value $O_{\text {sat }}$ as $t \rightarrow \infty$. As specific examples, we will discuss the mean-square displacement and the average number of sites covered by the random walker in time $t$. We propose that $O(p, L, t)$ satisfies the scaling law:

$$
O(p, L, t)=O_{\text {sat }} \mathcal{F}\left(p^{2} t, p L\right) .
$$

We have numerically confirmed this scaling Ansatz, as described in the rest of this paper. According to Eq. (1), a scaling collapse is observed if one plots the quantity of interest for various choices of the variables $(p, L)$, holding $x=p L=$ const., for any choice of $x$. This behavior is in contrast to previous statements that a scaling collapse cannot be expected when $x \gg 100$ [18]. Note also that the scaling collapse is seen only for fixed values of $x=p L$ and that a prerequisite for scaling is that $p \ll 1$. Previous

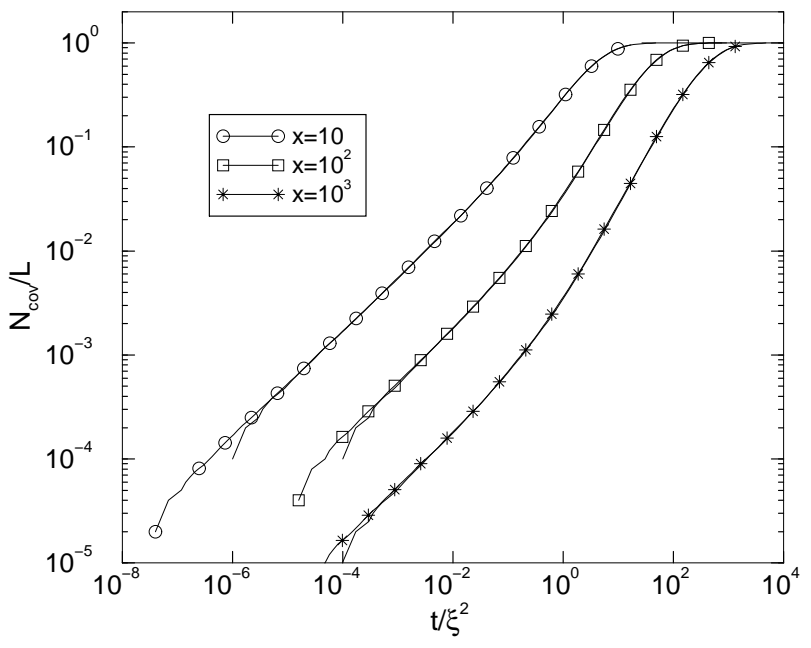

FIG. 1: This figure shows the scaling collapse for $N_{\text {cov }}$, the average number of distinct sites visited by the random walker, for $x=p L=10$ (circles, system sizes $L=10^{4}$ and $L=10^{5}$ ); $x=100$ (squares, $L=10^{4}$ and $2.5 \times 10^{4}$; and $x=1000$ (asterisks, $L=10^{5}$ and $L=2.5 \times 10^{5}$ ). The scaling collapse is very good.

workers 17, 18] have attempted to show scaling collapse for fixed values of $L$ (or $p$ ) while varying $p$ (or $L$ ), and did not obtain a perfect scaling collapse. By contrast, our present results display a perfect scaling collapse for fixed values of $x$. In the following, we present our numerical results for two quantities: the mean number of distinct sites covered, $N_{\text {cov }}$, and the mean square displacement, $\left\langle r^{2}\right\rangle$.

\section{B. Average Number of Distinct Visited Sites}

The average number of distinct nodes visited by a random walker, denoted $N_{\text {cov }}$, was first studied by Dvoretzky and Erdös [29] for a random walker on an infinite $d$ dimensional regular lattice. They found that $N_{\mathrm{COV}} \sim \sqrt{t}$ in 1-d, $N_{\mathrm{COV}} \sim t / \ln t$ in 2- $d$ and $N_{\mathrm{COV}} \sim t$ for $d>2$, in the limit of $t \rightarrow \infty$. How does the small-world network modify this result? Following the arguments of [17], we expect that for short times, $t \ll \xi^{2}$ (with $\xi=1 / p$ ), the random walker will be probing regions of the small-world network that are essentially without shortcuts (linear regions). Hence, the behavior should be similar to the $p=0$ case where the walker covers $N_{\text {Cov }} \sim \sqrt{t}$ sites. For long times, $t \gg L \xi$, we expect all the sites in the network to be covered and $N_{\mathrm{Cov}}=L$. For intermediate times, $\xi^{2} \ll t \ll L \xi$, the walker spends on the average $\xi^{2}$ time steps per linear region, before a new region is accessed - the shortcuts act like a kind of branching process for the random walk. As a consequence, in this time regime there should be $t / \xi^{2}$ segments covered and $N_{\mathrm{Cov}} \sim t[17]$. Combining these regimes, we obtain the scaling form

$$
N_{\mathrm{COV}}=L S\left(t / \xi^{2} ; x\right)
$$




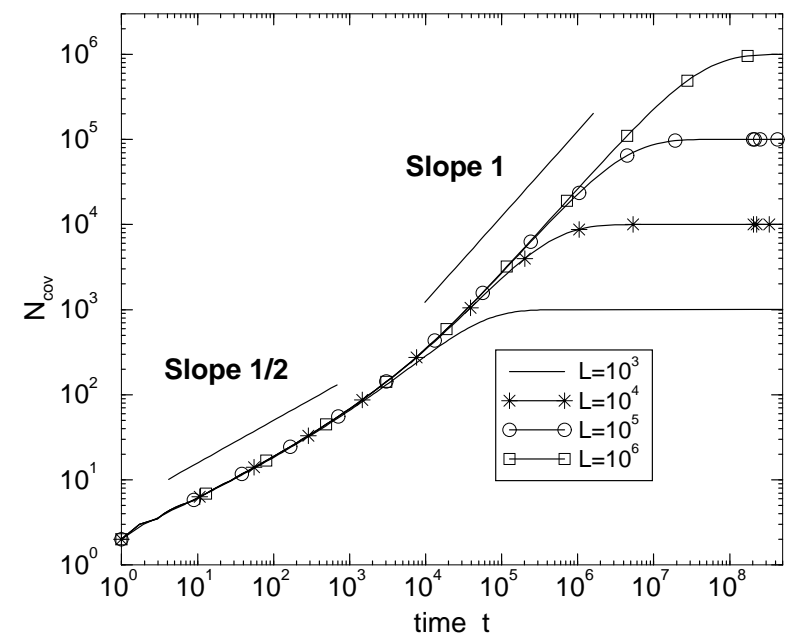

FIG. 2: Effects of finite size on the average number of covered sites, $N_{\text {cov }}$, which is plotted as a function of time $t$ at fixed density of shortcuts $p=0.01$, for four different system sizes: $L=10^{3}, 10^{4}, 10^{5}$ and $10^{6}$. The saturation seen at all four values of $L$ is a finite-size effect.

where

$$
S(y ; x) \sim\left\{\begin{array}{cc}
\sqrt{y} / x, & y \ll 1 \\
y / x, & 1 \ll y \ll x, \\
1, & y \gg x,
\end{array}\right.
$$

and $y=t / \xi^{2}$. This is the expected finite-size scaling form for the average number of distinct visited nodes in the small-world model in the limit $x>1$.

In Fig. 11 we show a plot of our calculated $N_{\mathrm{COV}}$ for $x=10$ (circles), $10^{2}$ (squares) and $10^{3}$ (stars). For each value of $x$, we have used different values of $p$, ranging from $p=0.0002$ to $p=0.01$, two for each $x$-value. The scaling collapse is excellent. These results suggest that the scaling properties of the small-world network determine the scaling of the random walk; hence, to find a scaling collapse, one must keep the average number of shortcuts, $x$, constant. This Figure also shows that, as $x$ increases, $N_{\text {cov }}$ deviates more and more from the $p=0$ result.

If, instead, we hold the density of shortcuts constant and vary the system size, we can explore the finite-size effects on the average number of covered sites, $N_{\text {cov }}$. In Fig. 2] we plot the succession of curves $L=10^{3}, 10^{4}, 10^{5}$ and $10^{6}$, all with $p=0.01$. Upon examination, we find that the slope of the resulting scaling curve changes from $1 / 2$ to 1 . That is, for small $t\left(t \ll \xi^{2}\right), N_{\mathrm{COV}} \sim \sqrt{t}$, after which, at larger $t$, there is a crossover to $N_{\text {cov }} \sim t$, and finally, at even larger $t$, finite-size effects become apparent. This behavior observed in the simulations agrees very well with the arguments preceding Eq. (3). Note that, when $\xi \lesssim L$, we do not see a crossover to the linear regime in which $N_{\text {cov }} \sim t$ before finite size effects start to become dominant.

We now derive an approximate expression for $N_{\mathrm{COV}}$ which is consistent with Eq. (3) and also in good agree-

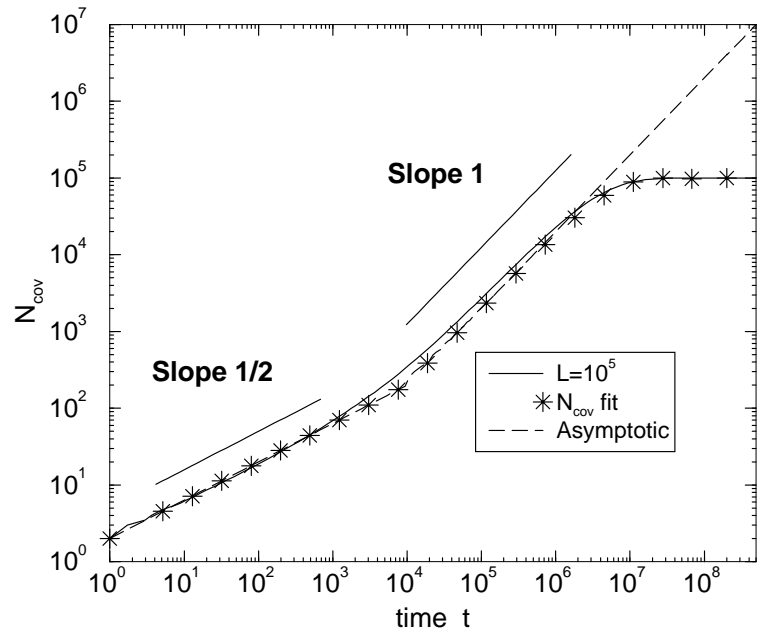

FIG. 3: Comparison of the approximation (stars) to $N_{\text {Cov }}$, Eq. (5), with numerical data (solid line) for $L=10^{5}$ and $p=$ 0.01 . The asymptotic $(L \rightarrow \infty)$ curve (dotted line) satisfies $N_{\text {Cov }} \propto \sqrt{t}$ for small $t\left(t \ll \xi^{2}=1 / p^{2}\right)$ and $N_{\text {cov }} \propto t$ for large $t\left(t \gg \xi^{2}\right)$. The change of slope between the two behaviors occurs near $t \sim \xi^{2}$.

ment with the large- $x$ simulation results. We note that, on average, there are $2 x$ shortcut ends in the network, defining $2 x$ contiguous "regions" in the $1-d$ network. If a walker takes a shortcut (or "jump"), it transports the walker from one region to another, randomly chosen, region in the network. When the number of jumps $n_{j}$ is small compared to $2 x$, there is a high probability that the regions visited by the walker are "distinct" (as defined more precisely below). However, when $n_{j} \sim 2 x$, there is a high probability that some regions will be visited more than once. These multiple visits lead to saturation effects since the already-visited sites do not contribute to $N_{\text {cov }}$.

We now derive an expression for the mean number of distinct regions visited by the walker in $n_{j}$ jumps. Because each jump transports the walker from one region to another, randomly chosen region, the number of distinct regions is obtained by solving the following problem: If $n_{j}$ integers are chosen independently and randomly from the set $\{1,2, \ldots, 2 x\}$, what is the mean number of distinct integers chosen? This latter problem is easily solved: The probability that a given integer is not chosen in a given trial is $q=1-\frac{1}{2 x}$; hence, the probability that a given integer is not chosen in $n_{j}$ trials is $q^{n_{j}} \approx e^{-n_{j} /(2 x)}$ (where we have assumed $x \gg 1$ ). Thus, the mean number of distinct integers chosen after $n_{j}$ trials is

$$
I_{\mathrm{COV}}=2 x\left(1-e^{-\frac{n_{j}}{2 x}}\right) .
$$

For the original problem, the above expression gives us the number of distinct regions visited by the walker after $n_{j}$ jumps, and each distinct region visited by the walker corresponds to covering $L /(2 x)$ sites. Furthermore, after time $t$, the random walker has, on average, taken $n_{j}=$ $\left\lfloor 4 p^{2} t\right\rfloor$ jumps. Hence, the probability that the walker 


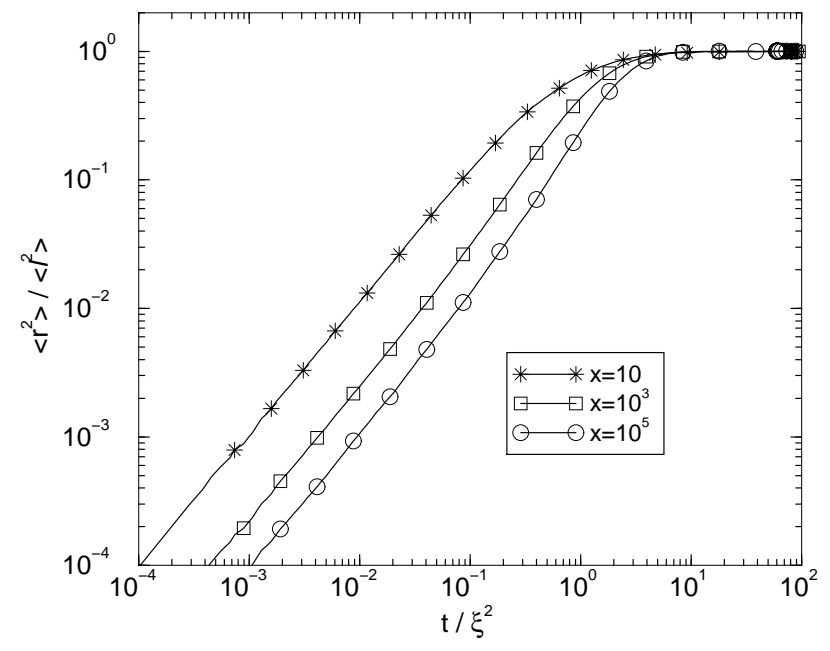

FIG. 4: Mean-square displacement, $\left\langle r^{2}(t)\right\rangle$, for a random walker on an SWN for several values of $p \leq 0.01$ and the three choices for average numbers of shortcuts: $x=p L=10$, $10^{3}$ and $10^{5}$. Note that each constant- $x$ curve consists of two distinct $(p, L)$ combinations. $\left\langle r^{2}(t)\right\rangle$ saturates at large $t$ because of finite-size effects.

visits a new region after the $n_{j}^{\text {th }}$ jump is $\exp (-\lfloor 2 y\rfloor / x)$. Also, the time spent by the walker in this new region is $t^{\prime}=t-\frac{1}{4 p^{2}}\left\lfloor 4 p^{2} t\right\rfloor$, while the number of sites covered in this time interval is given by $\sqrt{t^{\prime}}$, i.e. is proportional to the expression for the $p=0$ case. Combining these estimates, we find that the mean number of sites visited after time $t$ is given by

$$
S(y, x) \approx 1-e^{-\frac{2}{x}\lfloor y\rfloor}+\frac{2}{x} \sqrt{y-\lfloor y\rfloor} e^{-\frac{2}{x}\lfloor y\rfloor}
$$

Expression (15) is clearly only an approximation to the exact function $S(y, x)$. However, it does captures the key processes leading to the growth and saturation of $N_{\text {cov }}$ and hence is a useful approximation. In Fig. 3] we compare this function to the numerically obtained curves for $S(y, x)$ at $p=0.01$, taking $L=10^{5}$ and $L \rightarrow \infty$. The agreement is quite good and shows that Eq. (5) is a reasonable approximation to the scaling function.

\section{Mean-Square Displacement}

We now compute the mean-square displacement, $\left\langle r^{2}(t)\right\rangle$, of the random walker as a function of time $t$. To calculate this quantity, we first, at each time step, find the minimal distance from the current position of the random walker to the origin (i.e. the smallest number of steps needed for the random walker to reach the origin) using a breadth-first search method. Then we allow the walker to move through the network until $\left\langle r^{2}(t)\right\rangle$ has saturated. Finally, we average over different initial positions of the walker and realizations of the network.

Now, we know that for a random walk on an infinite, hypercubic, $d$-dimensional lattice $\left\langle r^{2}(t)\right\rangle=(1 / 2) D t$,

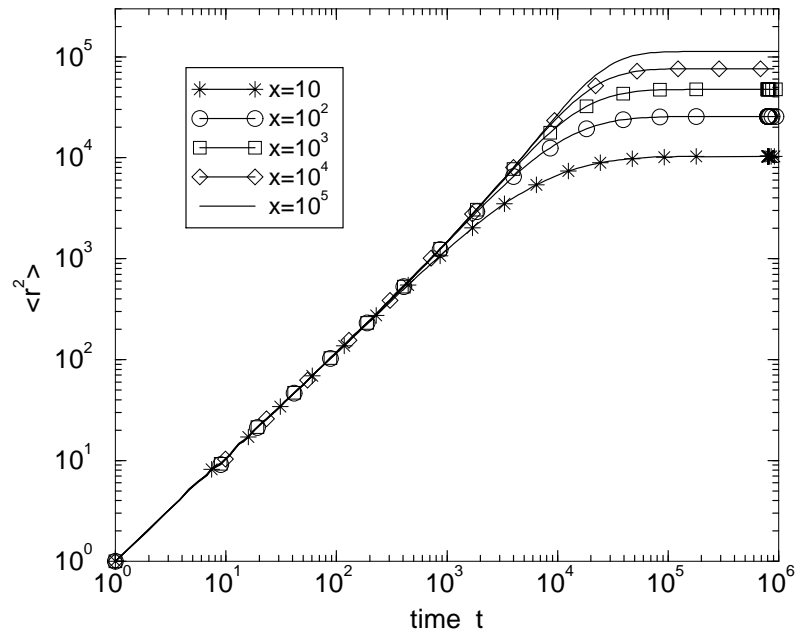

FIG. 5: Mean-square displacement, $\left\langle r^{2}(t)\right\rangle$, as a function of time $t$ for a random walker on a succession of networks, all with the same density of shortcuts $(p=0.01)$, and system sizes varying from $L=10^{3}$ to $L=10^{7}$. Except for very large $t$, all curves collapse onto a single scaling curve, the curve which would be obtained for $L \rightarrow \infty$, but at sufficiently large $t$, finite-size effects become important.

as can be shown using e.g. a generating function formalism [13, 28]. However, on a finite lattice, $\left\langle r^{2}(t)\right\rangle$, must approach a constant for large $t$. In this limit, each node of the network has equal probability of being occupied by the random walker. This insight immediately gives $\left\langle r^{2}\right\rangle=\overline{\ell^{2}}$, where $\overline{\ell^{2}}$ is the squared minimum distance between a pair of nodes, averaged over all possible pairs and network realizations.

On an SWN, the other relevant length scale for the random walker is $\xi=1 / p$, the average distance the walker travels to reach a shortcut. These two lengths suggest the following scaling Ansatz: $\left\langle r^{2}\right\rangle=\overline{\ell^{2}} R\left(t / \xi^{2} ; p L\right)$. We can also infer the behavior of $R(y, x)$ using simple arguments. For times $t \ll \xi^{2}$, the walker is exploring regions of the network without shortcuts, and we expect diffusive behavior similar to that of a regular network, giving $R(y, x) \sim y \overline{\ell^{2}}$. When $t \sim \sqrt{\overline{\ell^{2}}} / p$, we expect the mean-square displacement to saturate, and $R(y, x)=1$. The transition between the two types of behavior is not sharp, since the walker may reach a shortcut before it has travelled a distance $\xi$.

We have numerically confirmed this scaling collapse for a wide range of $x$ values. In Fig. 4 we plot $R\left(t / \xi^{2} ; x\right)$ for a sequence of networks with $x=p L=10,10^{3}$ and $10^{5}$. For each constant- $x$ curve, we show use two distinct values of $p \leq 0.01$. We see that initially, the walker probes a regular network, and $R\left(t / \xi^{2} ; x\right)$ is linear in $t$. When $t / \xi^{2} \sim 1$, the walker begins to reach some shortcuts in the network, and there is a crossover to superdiffusive behavior. Finally, at still larger $t / \xi^{2}$, finite-size effects become important and $R\left(t / \xi^{2} ; x\right)$ saturates.

To further explore finite-size effects on the mean- 


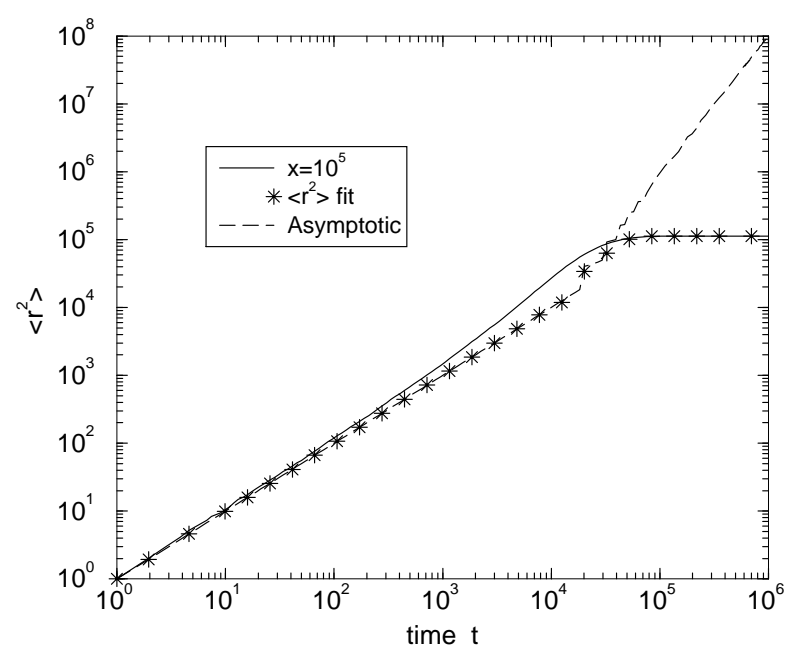

FIG. 6: Comparison of the approximation (stars) to $\left\langle r^{2}(t)\right\rangle$, Eq. (6), with numerical data (solid line) for $L=10^{7}$ and $p=$ 0.01 . The asymptotic $(L \rightarrow \infty)$ curve (dotted line) satisfies $\left\langle r^{2}(t)\right\rangle \propto t$ for small $t\left(t \ll \xi^{2}\right)$ and $\left\langle r^{2}(t)\right\rangle \propto t^{2}$ for large $t$ $\left(t \gg \xi^{2}\right)$.

square displacement, we have studied $\left\langle r^{2}(t)\right\rangle$ in a succession of networks, each with the same density of shortcuts, $p=0.01$, but with different linear size $L$. In Fig. 5] we plot the calculated $\left\langle r^{2}(t)\right\rangle$ for values of $L$ differing by factors of 10 and ranging from $10^{3}$ to $10^{7}$. The resulting curves are all very similar to the $L \rightarrow \infty$ curve, until finite size effects produce saturation with $\left\langle r^{2}\right\rangle=\overline{\ell^{2}}$. Only for the largest $x$-values are we able to reach the superdiffusive regime, and even for $x=10^{5}$, this regime is so narrow that we cannot with confidence determine the exponent of the expected power-law time dependence.

The development of an approximate analytical expression for $\left\langle r^{2}(t)\right\rangle$ is difficult, since we need the minimal distance between two lattice points. However, we know the limiting forms for $\left\langle r^{2}(t)\right\rangle$. Further, by making the approximation that the random walker only uses a short-cut once, we can extend the arguments used to derive the approximate expression for $N_{\mathrm{cov}}$, Eq. (5). Hence, we can write down the following ansatz for $\left\langle r^{2}(t)\right\rangle$ :

$$
R(y, x)=1-e^{-\frac{1}{p^{2} \overline{\ell^{2}}}\lfloor y\rfloor^{2}}\left(1-\frac{1}{p^{2} \overline{\ell^{2}}}(y-\lfloor y\rfloor)\right) .
$$

In Fig. 6] we compare this ansatz with the simulations for $L=10^{7}$; evidently it agrees reasonably well with the numerical results.

We can write down the scaling function in several limiting regimes, without necessarily using the above ansatz for the specific functional form. For example, we must have

$$
R(y ; x) \sim y /\left(p^{2} \overline{\ell^{2}}\right), \quad y \ll 1
$$

and

$$
R(y ; x)=1, \quad y \gg p \sqrt{\overline{\ell^{2}}}
$$

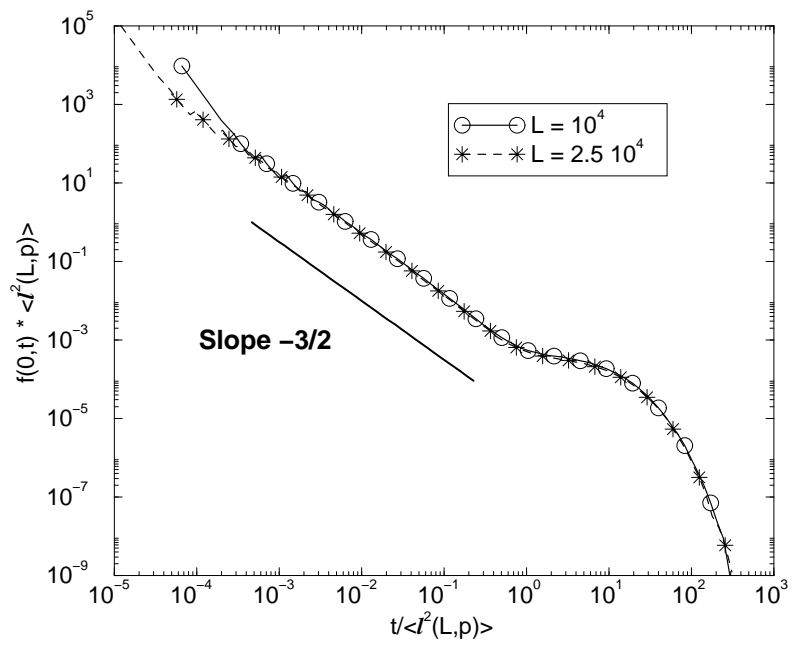

FIG. 7: Scaling collapse of the first-return time distribution, $f(0, t)$ plotted against the dimensionless time $u=t / \overline{\ell^{2}}(L, p)$ for networks with the average number of shortcuts $x=p L=$ 100 using $L=10^{4}$ and $L=2.5 \times 10^{4}$ nodes.

However, the known limiting behaviors cannot give the scaling function in the intermediate regime. We therefore use the above ansatz to propose

$$
R(y ; x) \sim y^{2} /\left(p^{2} \overline{\ell^{2}}\right), \quad 1 \ll y \ll p \sqrt{\overline{\ell^{2}}} .
$$

We are not able to verify this last behavior numerically from our present results.

\section{First Return Time Distribution}

Next, we turn to another property of a walker on an SWN, namely the distribution of first passage (or first return) times. This is the probability that a walker will return to a given site $m$ for the first time a time $t$ after leaving that site. We denote this distribution $f(m, t ; L, p)$. Note, that this distribution does not saturate to a finite value when $t \rightarrow \infty$, and hence, it shows that the scaling collapse of random walk properties is not only limited to those which saturate to a finite value. In order to find a smooth distribution, we must make a slight change to the random walker rules: For each time-step, the walker is now allowed to stay on the current node with probability $1 /(k(m)+1)$.

An extensive review of first passage processes has been given by Redner 31]. Unlike the other quantities presented in this article, the first-return time does not saturate to a finite value, instead it approaches zero. However, the scaling collapse is still present. This collapse is demonstrated in Fig. 7 where we plot $f(0, t)$ for two different $(p, L)$ combinations, holding $x$ constant.

The "small-world" effect on the first-return time distribution is most clearly seen at large values of $x$. In Fig. 8 we plot $f(0, t)$ for a network of $L=10^{6}$ sites and a shortcut density of $p=0.01$. For short waiting times, as 


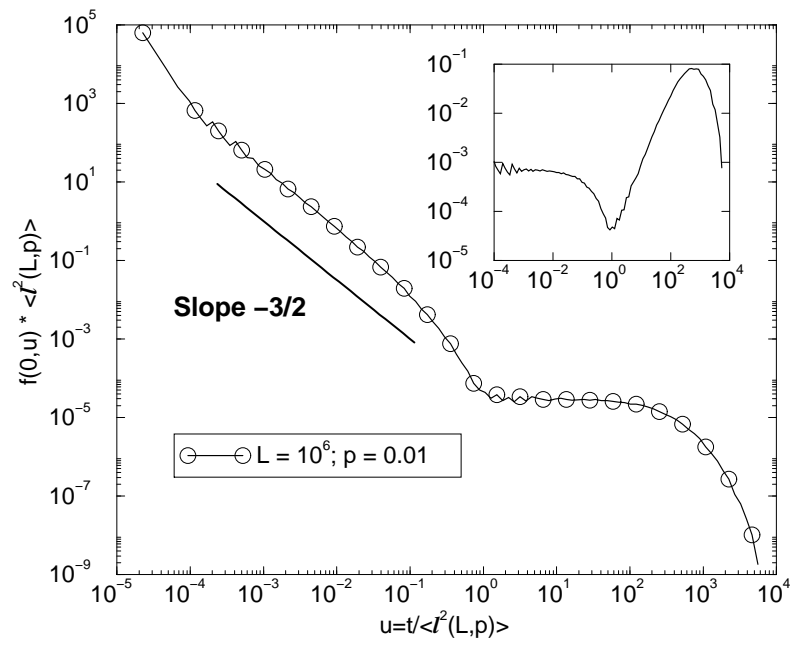

FIG. 8: First-return time distribution, $f(0, t)$, plotted against the dimensionless time $u=t / \overline{\ell^{2}}(L, p)$. For waiting times $t \ll \overline{\ell^{2}}(L, p)$, the first-return time distribution $f(0, t)$ behaves like that of a $p=0$ network, with $f(0, t) \sim t^{-3 / 2}$. For intermediate waiting times $\left(\overline{\ell^{2}}(L, p) \ll t \ll L \xi\right), f(0, t)$ is independent of $t$, since each node is occupied with equal probability. For $t>L \xi$, lattice finite-size effects dominate. The inset shows $\left(t / \overline{\ell^{2}}\right)^{3 / 2} f\left(0, t / \overline{\ell^{2}}\right)$.

expected, $f(0, t)$ behaves like that of a $p=0$ network (no shortcuts). However, for intermediate waiting times such that $\overline{\ell^{2}} \ll t \ll L \xi$, we find that $f(0, t)$ is independent of $t$. This independence indicates that the position of the random walker is completely randomized: the memory of the walker's starting position is no longer retained in the system and the walker is equally likely to occupy any site in the network. This occurs when $t \sim \overline{\ell^{2}}$, as discussed above for $\left\langle r^{2}(t)\right\rangle$. Also, $L \xi$ is the characteristic time for the asymptotic decay of $f(m, t ; L, p)$, since it is the saturation timescale for $N_{\text {cov }}$. Note that the separation of $\overline{\ell^{2}}(L, p)$ and $L \xi$ increases with increasing number of shortcuts $x$ in the system, making the "knee" of the first-return distribution more pronounced.

For a random walk on a finite interval $[0, L]$ with an absorbing wall at zero and a reflecting wall at $L$ there is a similar effect in the first return time 31, 32]; at $t \sim L^{2}$ there is an enhancement of the first return probability. In our case, the origin of this effect is the splitting of the timescales $\overline{\ell^{2}}$ and $L \xi$, while for the walk on the finite interval the cause for this effect are the contributions from reflected trajectories.

\section{SUMMARY}

In summary, we have studied the behavior of a random walker on a small-world network, using a combination of numerical methods and scaling assumptions. We conjecture that the scaling law of Eq. (1) is obeyed by measurable, saturating properties $\mathcal{O}(p, L, t)$ of a random walk on an SWN. Among these properties are the average number of distinct visited sites, $N_{\mathrm{Cov}}$, and the mean-square displacement $\left\langle r^{2}\right\rangle$ for random walks on SWN's, both of which we have studied over a wide range of node numbers $L$ and shortcut densities $p$. In both cases, we find that Eq. (11) is satisfied, and the quantities depend only on the single variable $x=p L$. Additionally, we find that non-saturating properties also show a scaling collapse, as exemplified by the first-return time.

Thus, we have shown that the dynamical behavior of a random walker on an SWN has the same scaling behavior as that exhibited by purely geometrical properties of the network (as described in e.g. Ref. [22]). This scaling behavior should be useful in interpreting a variety of other properties on SWN's, and may be of value in studying real-world phenomena for which an SWN is a good model.

\section{Acknowledgments}

This work has been supported by NSF through Grant No. DMR01-04987 (E.A. and D.S.) and the U.S. Department of Energy, Office of Science, Division of Materials Research (R.V.K).
[§] Current Address: NEC Laboratories, 4 Independence Way, Princeton, NJ 08540.

[1] S. H. Strogatz. Nature, 410, 268 (2001).

[2] R. Albert and A. L. Barabási. Rev. Mod. Phys., 74, 47 (2002).

[3] M. Kuperman and G. Abramson. Phys. Rev. Lett, 86, 2909 (2001).

[4] M. E. J. Newman. Phys. Rev. E, 66, 016128 (2002).

[5] R. Pastor-Satorras and A. Vespignani. Phys. Rev. Lett., 86, 3200 (2001).

[6] Z. Dezso and A.-L. Barabàsi. Phys. Rev. E, 65, 055103 (2002).

[7] F. Liljeros, C. R. Edling, L. A. N. Amaral, H. E. Stanley, and Y. Aberg. Nature, 411, 907 (2001).
[8] L. F. Lago-Fernandez, R. Huerta, F. Corbacho, and J. A. Siguenza. Phys. Rev. Lett., 84, 2758 (2001).

[9] R. Albert and A.-L. Barabàsi. Phys. Rev. Lett., 84, 5660 (2002).

[10] M. Barahona and L. M. Pecora. Phys. Rev. Lett., 89, 054101 (2002).

[11] S. N. Dorogovtsev and J. F. F. Mendes. Adv. Phys., 51, 1079 (2002).

[12] A. Scala, L. A. N. Amaral, and M. Barthélémy. Europhys. Lett., 55, 594 (2001).

[13] M. N. Barber and B. W. Ninham. Random and Restricted Walks. Gordon and Breach, New York, 1970.

[14] B. D. Hughes. Random Walks and Random Environments, volume 1 and 2. Clarendon Press, Oxford, 1996. 
[15] R. Monasson. Eur. Phys. J. B, 12, 555 (1999).

[16] S. Jespersen, I. M. Sokolov, and A. Blumen. Phys. Rev. E, 62, 4405 (2000).

[17] F. Jasch and A. Blumen. Phys. Rev. E, 63, 041108 (2001).

[18] J. Lahtinen, J. Kertész, and K. Kaski. Phys. Rev. E, 64, 057105 (2001).

[19] S. A. Pandit and R. E. Amritkar. Phys. Rev. E, 63, 041104 (2001).

[20] B. Luque and O. Miramontes. preprint cond-mat/0211383 November 2002.

[21] J. Lahtinen, J. Kertész, and K. Kaski. Physica A 311, 571 (2002).

[22] R. V. Kulkarni, E. Almaas and D. Stroud. Phys. Rev. E, 61, 4268 (2000).

[23] E. Almaas, R. V. Kulkarni, and D. Stroud, Phys. Rev. Lett 88, 098101 (2002).

[24] D. J. Watts and S. H. Strogatz. Nature, 393, 440 (1998).

[25] M. E. J. Newman and D. J. Watts. Phys. Lett. A, 263, 341 (1999).
[26] M. Molloy and B. Reed. Random Struct. Algorithms, 6, 161 (1995).

[27] M. E. J. Newman and S. H. Strogatz and D. J. Watts. Phys. Rev. E, 64, 026118 (2001).

[28] E. W. Montroll. Random walks on lattices. In R. Bellmann, editor, Stochastic Processes in Mathematical Physics and Engineering, volume 16 of Proceedings of Symposia in Applied Mathematics, pages 193-220. American Mathematical Society, Rhode Island, 1964.

[29] A. Dvoretzky and P. Erdös. Some problems on random walk in space. In Proc. 2nd Berkeley Sympos. Math. Statist. and Prob., pages 353-367. Univ. of California Press, Berkeley, Calif., 1950.

[30] M. E. J. Newman and D. J. Watts. Phys. Rev. E, 60, 7332 (1999).

[31] S. Redner. A guide to first-passage processes. Cambridge Univ. Press, New York, 2001.

[32] S. Redner. Private communication. 